\title{
Efectos en los sistemas de salud de la pandemia por COVID-19
}

\section{Effects on health systems of the COVID-19 pandemic}

\author{
Johana Acosta-Quiroz ${ }^{1 a}$, Sebastián Iglesias-Osores, ${ }^{1 a}$
}

\section{Sr. Editor:}

Los efectos de la pandemia causada por el coronavirus SARS-CoV-2 en los sistemas de salud destacan el papel de la Organización Mundial de la Salud (OMS) y gobiernos de diferentes países para contener la enfermedad. En diciembre de 2019 se reportó el brote de una neumonía de causa desconocida en Wuhan, China. Las autoridades sanitarias de dicho país identificaron mediante secuenciación que se trataba de un nuevo coronavirus $(2019-n \mathrm{CoV}){ }^{(1)}$. En China se produjo un retraso en detectar la enfermedad lo que llevó a un colapso en el sistema de salud ${ }^{(2)}$. Los brotes de enfermedades infecciosas requieren que se tomen medidas coordinadas de prevención en salud pública ${ }^{(3)}$. Las medidas de salud pública como la cuarentena, el distanciamiento social y la rápida detección de casos son acciones que tratan de evitar la propagación de la enfermedad ${ }^{(2)}$. El objetivo de esta carta es comentar algunos aspectos de los efectos originados por la COVID-19 en los sistemas sanitarios.

Esta pandemia está poniendo en evidencia las deficiencias en el sector salud alrededor del mundo como la escasez de equipos de protección personal, de ventiladores mecánicos para aquellos que necesitan atención médica e inclusive deficiencias en la recolección de muestras y pruebas para diagnosticar la infección. La COVID-19 se transmite fácilmente de persona a persona y aún no hay un medicamento efectivo para combatirlo. Las medidas de prevención más eficaces son las siguientes: ubicar: Pacientes sospechosos y personas cercanas, confirmar casos y evitar la transmisión por medio del aislamiento, desinfección y protección personal.

Reducir el contacto entre personas disminuye la transmisión del virus en una comunidad y ayuda a que los sistemas de salud respondan de manera eficaz. El enfoque más importante es quedarse en casa y salir solo para la compra de productos de primera necesidad y necesidades médicas. Además proteger la salud del personal de salud es primordial para el correcto funcionamiento del sistema de salud (4).

La capacidad del sector salud será rebasado rápidamente a medida que la propagación del virus aumente, las estrategias de salud pública deben estar enfocadas en aumentar el número de camas, ventiladores y equipos de protección personal ${ }^{(4)}$. La educación sanitaria es crucial para prevención y control de enfermedades, así como la detección de la mismas. El aislamiento y tratamiento de los pacientes son puntos clave para la prevención y reducción de las tasas infección ${ }^{(5)}$. Algunas de las claves que se podría implementar en los sistemas sanitarios son:

1. Fortalecer el sistema de diagnóstico molecular a nivel nacional, favoreciendo a los laboratorios ya instalados en instituciones públicas o privadas.

2. Crear $\mathrm{UCl}$ con áreas separadas del resto de las camas de $\mathrm{UCl}$ para minimizar el riesgo de transmisión en el hospital.

\footnotetext{
1. Facultad de Ciencias Biológicas Hospital Regional Lambayeque. Chiclayo, Perú.

a. Biólogo
} 
3. Organizar un área de triaje donde los pacientes puedan recibir ventilación mecánica si es necesario mientras se espera del resultado final de las pruebas de diagnóstico.

4. Establecer protocolos locales para la clasificación de pacientes con síntomas respiratorios, para evaluarlos rápidamente y, según el diagnóstico, asignarlos al servicio apropiado.

5. Asegurarse de que el equipo de protección personal (EPP) para los profesionales de la salud esté disponible mediante la organización de un suministro y la distribución adecuados, y la capacitación a todo el personal en riesgo de contagio.

6. Reemplazo rápido y oportuno de las bajas en el personal sanitario por ser de riesgo o haberse contagiado.

7. Mejorar el sistema de suministro de oxígeno medicinal a nivel nacional, incrementar la producción y el acceso a sistemas de oxígeno medicinal.

Los sistemas sanitarios y las políticas públicas sanitarias en los países en desarrollo como los latinoamericanos son muy frágiles, pues en esta pandemia estamos a punto de vernos saturados por el número y complejidad de los casos atendidos. La problemática incluye desde el mismo diseño de la infraestructura hasta el abastecimiento de material médico, pasando por la falta de personal sanitario. Esperamos que esta crisis sea una oportunidad de mejora para todo el sistema sanitario de países latinoamericanos, asi como la capacidad de manejo en emergencias.

Fuentes de financiamiento: Autofinanciado.

Conflicto de intereses: Ninguno

\section{REFERENCIAS BIBLIOGRÁFICAS}

1. Zhu N, Zhang D, Wang W, et al. A novel coronavirus from patients with pneumonia in China, 2019. N Engl J Med. 2020;382(8):727-733. doi:10.1056/ NEJMoa2001017

2. Khanna RC, Cicinelli MV, Gilbert SS, Honavar SG, Murthy GS V. COVID-19 pandemic: Lessons learned and future directions. Indian $\mathrm{J}$ Ophthalmol. 2020;68(5):703-710. doi:10.4103/ijo.IJO_843_20

3. Burkle FM. Declining Public Health Protections within Autocratic Regimes: Impact on Global Public Health Security, Infectious Disease Outbreaks, Epidemics, and Pandemics. Prehosp Disaster Med. Published online April 2, 2020:1-38. doi:10.1017/s1049023×20000424

4. Guest JL, del Rio C, Sanchez T. The 3 Steps Needed to End the COVID-19 Pandemic: Bold Public Health Leadership, Rapid Innovations, and Courageous Political Will (Preprint). JMIR Public Heal Surveill. 2020;6(2). doi:10.2196/19043

5. Deng S-Q, Peng H-J. Characteristics of and Public Health Responses to the Coronavirus Disease 2019 Outbreak in China. J Clin Med. 2020;9(2):575. doi:10.3390/jcm9020575. 